\section{Anesthesia for Cesarean Delivery in the Morbidly Obese Parturient - Comparison of Commonly used Anesthesia Techniques}

Ali S Faris ${ }^{1 *}$, Alan J Chaput ${ }^{2}$, Susan Goheen ${ }^{2}$ and Catherine Gallant $^{2}$

${ }^{1}$ Department of Anesthesiology and Pain Management, Tawam Hospital, Al Ain, UAE

${ }^{2}$ Department of Anesthesiology, The Ottawa Hospital, Ottawa, Ontario, Canada

\begin{abstract}
Background: Obesity in pregnancy can add significantly to obstetric and anesthetic complications. The objective of this study is to identify and compare anesthesia-related complications in different types of elective cesarean anesthetics in morbidly obese parturients.

Methods: Retrospective review was done on 205 records of elective cesareans for pregnants with Body Mass Index (BMI) $\geq 40$. Data extracted include: type of anesthesia, intraoperative and early postoperative complications, duration of surgery, newborn birth weight, cord blood pH and Apgar scores.

Results: The incidence of significant intra-operative hypotension: $65.0 \%$, bradycardia $3.04 \%$, nausea and vomiting $14.21 \%$. Intraoperative hypotension: $67 \%$ in spinal and CSE group vs. $34.8 \%$ in epidural group $(p=0.0022)$. Super Morbid Obese $(S M O)$ patients $(\mathrm{BMI}>50)$ had a greater number of attempts at placement for regional techniques compared to Morbidly Obese (MO) patients (BMI 40-49.9) (2.02 vs. 2.61, $p=0.0255)$. SMO patients had longer mean surgical times (86.6 vs. $107.55 \mathrm{~min}, \mathrm{p}=0.0001$ ), received more intraoperative crystalloids (1887 vs. $1699 \mathrm{ml}$, $p=0.0376)$ and colloids (33.9 vs. $12.6 \%, p=0.0007$ ) compared to MO patients. No other complications were significantly different between the 2 compared anesthetic techniques or BMI classes.

Conclusion: Spinal anesthesia was the most commonly used technique. It resulted in more hypotension. Parturients with $\mathrm{BMI} \geq 50$ were more challenging to manage with more regional
\end{abstract}

*Corresponding author: Ali S Faris, Department of Anesthesiology and Pain Management, Tawam Hospital, PO Box 64024, Al Ain, UAE, Tel: +971 37649964; E-mail: alfaris@hotmail.ca

Citation: Faris AS, Chaput AJ, Goheen S, Gallant C (2014) Anesthesia for Cesarean Delivery in the Morbidly Obese Parturient - Comparison of Commonly used Anesthesia Techniques. J Anesth Clin Care 1: 002.

Received: September 23, 2014; Accepted: November 24, 2014; Published: December 08, 2014 anesthesia placement attempts, larger fluid volumes and longer surgery times. A well-organized RCT could prove beneficial in validating the most profitable and safe anesthetic technique for bariatric parturients.

\section{Introduction}

Obesity is a worldwide growing healthcare problem affecting women of childbearing age. This was clearly identified in the World Health Organization (WHO) consultation report which classified obesity based on Body Mass Index (BMI) and related this to risk of comorbidities (Table 1) [1-4]. In this classification system, a BMI greater than 29.9 was defined as obese. Furthermore, the American National Heart, Lung, and Blood Institute (NHLBI) classified persons with $\mathrm{BMI} \geq 40$ as extremely or morbidly obese. Many studies have shown the increasing prevalence of obesity among adults to more than double in the past three decades, and that obesity continues to be a public health concern. In fact, in 2007-2008 about one third of the adult population in the United States had BMI of 30.0 or more [5-7].

\begin{tabular}{|c|c|c|}
\hline Class & BMI $\left(\right.$ Kg. $\left.^{-\mathbf{2}}\right)$ & Risk of Comorbidities \\
\hline Normal & $18.5-24.9$ & Medium \\
\hline Overweight & $\geq 25$ & Increased \\
\hline Pre-obese & $25-29.9$ & Moderate \\
\hline Obese class 1 & $30-34.9$ & Severe \\
\hline Obese class 2 & $35-39.9$ & Very severe \\
\hline Obese class 3 & $\geq 40$ & \\
\hline
\end{tabular}

Table 1: WHO Classification of Obesity.

The dramatic rise in the rate of obesity recorded in general population also involves women of reproductive age. In the United States, pre-pregnancy obesity was found to increase from 13\% in $1993-1994$ to $22 \%$ in $2002-2003$ in a study looking at trends in pre-pregnancy obesity [8].

Obesity is occurring with increasing frequency during pregnancy, such that in the last decade, maternal and fetal risks are being qualified, quantified and documented. It is well known that any bariatric patient is at risk for diseases such as diabetes, hypertension, respiratory diseases (including obstructive sleep apnea), and deep venous thrombosis, in addition to surgical complications such as wound infection [1]. If pregnancy is superimposed on being significantly overweight, patients become more prone to the development of antenatal diseases including pre-eclampsia, gestational diabetes, venous thrombo-embolism, large for gestational age infants, and preterm deliveries $[4,9,10]$.

The objective of this study was to identify the types of anesthesia that were employed for elective cesarean deliveries in morbidly obese parturients (BMI $\geq 40$ ) in our institution. In doing so, we sought to illustrate potential anesthesia-related adverse effects occurring during cesarean delivery for this patient cohort, and to discern whether there is a safer and more proficient anesthetic technique for this unique patient population. 


\section{Methods}

This study was approved by the Ottawa Hospital Research Ethics Board (OHREB) - protocol \#2011157-01H. In this retrospective cohort study, we included pregnant women with $\mathrm{BMI} \geqq 40$ at delivery time, who delivered their infants by planned cesarean section between Jan 1, 2007 and January 31, 2011 at the Ottawa Hospital. The Ottawa Hospital (Civic and General Campuses) is an academic tertiary care centre in Ottawa, Ontario, Canada, with a delivery rate of $\sim 7,000$ per year. Emergency cesarean deliveries were excluded. In order to identify parturients fitting our inclusion criteria, approval was granted, by the regional coordinator, for information access to Niday perinatal database, which is Ontario's perinatal database website. Once found, the pertinent patient charts were accessed from the Ottawa Hospital Medical Records Department and reviewed, by a single investigator (A.S.F.) to collect the required study data.

Maternal clinical characteristics that were extracted from the charts included: age, body weight, height, delivery BMI, American Society of Anesthesiologists (ASA) physical classification class, co-morbidities and current medications. Data related to the anesthetic technique that was extracted included: type of anesthesia, number of attempts required for successful regional anesthetic techniques, needle types used including the needle length and gauge, use of ultrasound to aid in regional anesthesia placement. In addition, information related to the duration of surgery, estimated blood loss and total amount and types of intra-operative fluids were recorded.

The first outcome measure was the composite intraoperative and early postoperative (in Post-Anesthesia Care Unit-PACU) anesthesia related adverse effects which included: incidence and duration of significant hypotension (defined as Systolic Blood Pressure -SBP $<25 \%$ of baseline or $\geq 90 \mathrm{mmHg}$ ), along with the treatment required (if any), specifying type and dose of vasopressors and/or intravenous fluids administered. Also, the incidence of maternal bradycardia and type and doses of anticholinergic medications if used, incidence of nausea and vomiting, with the type and doses of antiemetic medications if used.

Other outcome measures included the length of stay in PACU and the frequency of the need for additional intraoperative and early postoperative (in PACU) Intravenous (IV) analgesia, other than our routine acetaminophen and ibuprofen adjuvants. Also, data linked to the fetal outcome were evaluated including: baby's birth weight, Apgar scores at 1 and 5 minutes and fetal umbilical cord arterial blood $\mathrm{pH}$. We compared between the spinal and epidural groups of patients in terms of the incidence of intra- and early post-operative complications. Also, we divided our studied group of patients into according to the delivery BMI, into 2 groups: parturients with BMI 40-49.9 (morbidly obese) and the group of parturients with BMI of 50 or more (super morbidly obese). A comparison was made between these two groups of patients regarding all the collected data.

All categorical variables were compared by $x^{2}$ test or Fisher's exact test where appropriate. All continuous variables were compared using the independent $t$ test. SAS 9.2 was applied to conduct the statistical analysis.

\section{Results}

Two hundred and five medical records, for patients who have fulfilled our inclusion criteria, were reviewed. The mean age and anthropometric data of patients can be seen in Table 2 .

\begin{tabular}{|c|c|c|c|c|}
\hline Minimum & Maximum & Median & Mean & N \\
\hline 17 & 46 & 33 & 32.2 & 205 \\
\hline 82 & 206 & 122 & 125.1 & 205 \\
\hline 125 & 181 & 164 & 164.1 & 205 \\
\hline 40 & 71 & 46 & 47 & 205 \\
\hline
\end{tabular}

Table 2: Age and Anthropometric Data.

Of the 205 patient who were reviewed, 58 patients (28\%) were ASA class II and 146 patients were ASA class III (71\%) while only 1 patient was ASA class IV (1\%), see Table 3 . Also, it can be seen in table 3 that significant proportion of women had comorbidities including diabetes mellitus, hypertension and asthma. In addition, 12 parturients had multiple pregnancy and 3 had placenta previa.

\begin{tabular}{|c|c|}
\hline ASA Class & Number (\%) \\
\hline II & $58(28.3)$ \\
\hline III & $146(71.2)$ \\
\hline IV & $1(0.5)$ \\
\hline Co-morbidities & Number (\%) \\
\hline Hypertension & $42(20.5)$ \\
\hline Diabetes Mellitus & $36(17.6)$ \\
\hline Asthma & $24(11.7)$ \\
\hline Hypothyroidism & $14(6.8)$ \\
\hline Obstructive Sleep Apnea & $6(2.9)$ \\
\hline DVT & $4(2.0)$ \\
\hline Scoliosis & $3(1.5)$ \\
\hline Others* & $34(16.6)$ \\
\hline Multiple Pregnancy & $12(5.9)$ \\
\hline Placenta Previa & $3(1.5)$ \\
\hline
\end{tabular}

Table 3: Patient's ASA Classes \& Co-morbidities.

*Others: includes SVT, Cardiomyopathy, Aortic dilatation, Thrombocytopenia, Thalassemia, Coagulation disorders, Depression, Anxiety, Bipolar disorder, Substance abuse, Pituitary tumour, Bell's palsy, Kidney disease, Fatty liver disease, Muscle disease and Pelvic inflammatory disease.

$100(49 \%)$ of our reviewed patients had no previous cesarean sections and $84(41 \%)$ had 1 previous cesarean section, while $18(9 \%)$ of them had 2 previous cesarean sections and $2(1 \%)$ had 3 previous cesarean sections, whereas only 1 had 4 previous cesarean deliveries.

Anesthetics were conducted by the attending staff anesthesiologist or senior anesthesia resident under supervision of the staff anesthesiologist. Spinal anesthesia was given in 151 of the reviewed cases $(74 \%)$ and epidural anesthesia was the anesthetic technique in 26 parturients (13\%). Combined Spinal-Epidural (CSE) was given in 20 parturients (10\%) - usual spinal doses were given while epidural was used only to extend the block if needed, while general anesthesia was needed to be given in 8 parturients $(4 \%)$ due to difficulty to put the spinal or total patient refusal to neuraxial block. Bupivacaine $0.75 \%$ was the only local anesthetic used in the spinal anesthesia (dose $11 \mathrm{mg} \mp 1.5)$ in addition to fentanyl $(10-25 \mathrm{mcg}$ ) and preservative-free morphine $(50-200 \mathrm{mcg})$.

Statistical analysis of fetal data is presented in Table 4.

The incidence of significant intra-operative hypotension after regional anesthesia in our patient's cohort was 65\% (128 patients) while the incidence of bradycardia requiring treatment with anticholinergic medications was $3 \%$ (6 patients). The incidence of 


\begin{tabular}{|c|c|c|c|c|c|c|c|c|}
\hline Variable & N & Mean & Standard Deviation & Minimum & Lower Quartile & Median & Upper Quartile & Maximum \\
\hline Baby birth weight G & 220 & 3465.5 & 754.97 & 551 & 3074 & 3480.5 & 3993.5 & 5654 \\
\hline Apgar 1 & 220 & 7.77 & 1.89 & 0 & 7 & 9 & 9 & 10 \\
\hline Apgar 5 & 220 & 8.83 & 0.8 & 0 & 9 & 9 & 9 & 10 \\
\hline Cord pH & 220 & 7.23 & 0.15 & 6.91 & 7.18 & 7.24 & 7.27 & 7.4 \\
\hline
\end{tabular}

Table 4: Babies Birth Weight, Apgar Scores and Cord blood pH.

intra-operative nausea and vomiting was $14 \%$ (28 patients). Supplemental intra-operative IV analgesia was required in 13\% (24 patients) of the patients who had regional anesthetic techniques. Extra-length needles - $120 \mathrm{~mm}$ (Spinal or epidural) were required in $27 \%$ (54 patients) of patients. There were three occasions where spinal anesthetic technique needed to be converted to general anesthesia due to wearing of spinal block effect.

Significant postoperative hypotension was detected in 10\% (20 patients) of patients; all of them were treated by giving crystalloid or colloid fluid boluses, while nausea and vomiting were present postoperatively in $17 \%$ (20 patients) of patients where Ondansetron was given. Despite our hospital's routine practice of giving acetaminophen and one NSAID's medication once the parturient in the PACU after cesarean section, these analgesics were not given in $34 \%$ (69 patients) of our patients and pain was treated with parenteral opioids. The mean stay time in the PACU was 2.3 hours.

\section{Spinal/CSE vs. epidural technique}

Comparison of the spinal and CSE techniques group with the epidural group demonstrated a significantly higher incidence of intra-operative hypotension in the first group (Spinal \& CSE) 107 patients $(67.7 \%)$ compared to 8 patients $(34.8 \%)$ in epidural group, p-value $=0.0022$ (Table 5$)$. There was, however, no observed statistically significant difference in other intra-operative complications such as bradycardia, nausea and vomiting, intra-operative fluids (crystalloids and colloids), and extra-analgesic requirements, Additionally, no significant differences were noted in post-operative complications including hypotension, nausea and vomiting, bradycardia and duration of PACU stay between the two groups. From a neonate perspective, no statistically significant differences were noted between the two groups in terms of fetal cord blood $\mathrm{pH}$ and APGAR scores at 1 and 5 minutes post-delivery.

\begin{tabular}{|c|c|c|c|c|}
\hline \multirow{2}{*}{ Type of Anesthesia } & \multicolumn{2}{|c|}{ Significant Hypotension } & \multirow{2}{*}{ Total } & \multirow{2}{*}{ P Value } \\
\cline { 2 - 3 } & No & Yes & & \\
\hline $\begin{array}{c}\text { Epidural } \\
\text { N (\%) }\end{array}$ & $\begin{array}{c}15 \\
65.22 \%\end{array}$ & $\begin{array}{c}8 \\
34.78 \%\end{array}$ & 23 & \multirow{2}{*}{0.0022} \\
\hline $\begin{array}{c}\text { Spinal \& CSE } \\
\text { N (\%) }\end{array}$ & $\begin{array}{c}51 \\
32.28 \%\end{array}$ & $\begin{array}{c}107 \\
67.72 \%\end{array}$ & 158 & \\
\hline Total (No.) & 66 & 115 & 181 & \\
\hline
\end{tabular}

Table 5: Significant Hypotension according to Anesthetic Technique.

\section{Comparison of two BMI groups}

The cohort was divided, according to the delivery BMI, into 2 groups: parturients with BMI 40-49.9 (morbidly obese) and the group of parturients with BMI of 50 or more (super morbidly obese). By comparing and analysing the data from the two groups, there was a significant difference in the number of attempts at needle placement for regional anesthesia, $\mathrm{p}$-value $=0.0255$. Also there was a statistically significant difference in the mean surgery time between the two groups, $\mathrm{p}$-value $<0.0001$. The amount of intra-operative crystalloid and colloid fluids was also significantly higher in the super morbidly obese group, p-value $=0.0007$ (Table 6).

\begin{tabular}{|l|c|c|c|}
\hline & $\begin{array}{c}\text { Morbidly Obese } \\
\text { (MO) } \\
\text { (BMI 40-49.9) }\end{array}$ & $\begin{array}{c}\text { Super Morbidly } \\
\text { Obese } \\
\text { (SMO) (BMI } \geq \mathbf{5 0})\end{array}$ & P value \\
\hline $\begin{array}{l}\text { Mean of No. of Needle } \\
\text { placement Attempts }\end{array}$ & 2.02 & 2.61 & 0.0255 \\
\hline Mean Surgical Time (min) & 86.6 & 107.55 & 0.0001 \\
\hline $\begin{array}{l}\text { Mean Intra-operative } \\
\text { Crystalloids volume (mI) }\end{array}$ & 1699 & 1887 & 0.0376 \\
\hline $\begin{array}{l}\text { Patients Required } \\
\text { Intra-operative Colloids (\%) }\end{array}$ & 12.6 & 33.9 & 0.0007 \\
\hline
\end{tabular}

Table 6: Comparison between Classes of Morbid Obesity - Significant Results.

In our statistical analysis we did not find significant differences in the intraoperative estimated blood loss, intra- and postoperative hypotension, bradycardia, nausea and vomiting or extra-analgesic requirements between morbidly obese and super morbidly obese patients. Additionally, the PACU stay time, post-operative fluids requirement, babies' birth weights, fetal cord blood $\mathrm{pH}$ and Apgar scores at 1 and 5 minutes were not significantly different between the two groups.

\section{Discussion}

Obesity is a multi-systemic disorder that results in physiological changes superimposed on the systemic functional changes of pregnant women. It can bring about increase in both maternal and perinatal morbidity and mortality [11]. Of the most substantially important changes that have implications on the anesthesia-related side effects are the cardio-pulmonary effects of obesity. Both oxygen consumption and carbon dioxide production have linear relationship with body weight. Because of the augmented fat mass in the chest and abdominal walls and the upward retraction of this adipose tissue caused by supine position, respiratory difficulty and even oxygen desaturation can be experienced by the obese pregnant woman as these factors further lower the functional residual capacity, expiratory and inspiratory reserve volumes and chest wall compliance leading to worsening of the V/Q relationship [12-14].

Obesity can cause further increase in the already raised cardiac output of the pregnant woman (normally reaches approximately $50 \%$ greater than the pre-pregnant volume in second \& third trimesters). On the other hand, obesity impairs the reduction in the afterload that is associated with pregnancy by increasing peripheral vascular resistance and greater conduit artery stiffness. This can lead to potentially decreased cardiac functional reserves in obese patients and puts the obese parturient at particular risk. Also, obesity has been mentioned as a risk factor for peripartum cardiomyopathy [15-18].

Obesity can add significantly to both obstetric and anesthetic associated morbidity [19]. Obese parturients have a tendency to labour abnormally, which may contribute to increased instrumental and caesarean delivery (2 to 4 fold increase) [9,10,20,21]. Considerable challenges are faced by anesthesiologists when helping 
to deliver these patients by caesarean: these patients are more likely to have technical challenges with airway access, ease of intubation, intravenous access, spinal or epidural placement, and analgesic/anesthetic success with regional techniques, not to mention prolonged operation times and difficulty with adequate left uterine displacement positioning on the operating table $[9,19,20,22,23]$.

Regional anesthesia (spinal or epidural) is the technique of choice when it comes to cesarean delivery. It is the anesthetic route proven to be the safest for the parturient while at the same time providing optimal post-operative pain control. This is true in both normal weight and bariatric parturients [24-27]. The first inference that can be made from our bariatric parturient study sample is that spinal anesthesia continues to be used more often than CSE and epidural anesthesia for caesarean sections even in the morbidly obese parturients. In our studied cohort of patients, regional anesthesia was the chosen anesthetic technique in $96 \%$ of the cases, while spinal anesthesia was preferred to be used in $74 \%$ of the cases.

One of the common and well-known side effects of spinal anesthesia however is hypotension (incidence between 53-65\%) $[27,28]$. We observed that the spinal anesthesia technique (spinal alone and the spinal component of a CSE) resulted in more intraoperative hypotension (77\%) compared to the epidural technique (35\%). The drop in blood pressure can be caused by either significant vasodilatation from the sympathetic blockade, or due to positioning inadequacies leading to aortocaval compression. In the normal weight patient, the blood pressure change can be usually compensated for quickly by minor position changes followed by fluid administration and vasopressor medications if necessary. Unfortunately, in the significantly overweight patient this complication can be much more challenging, which might be due to the difficulty to achieve the optimum positioning of the bariatric parturient quickly. In fact some studies even identified that obesity alone may be a risk factor for hypotension to develop after spinal anesthesia $[27,28]$. This is due to increased intra-abdominal fat and weight causing aortocaval compression with inherent decreased blood return to the heart. Slow titration of an epidural for these women may allow for better blood pressure compensation with fluid administration and repositioning before hypotension is experienced.

Additionally, maternal bradycardia can be concurrent with hypotension, both of which may lead to the deleterious side effects of intra-operative nausea, vomiting, and fetal academia $[27,29,30]$. The overall incidence of intraoperative bradycardia in our studied group of patients was $3.4 \%$ ( 7 patients), while 28 patients (13.66\%) experienced nausea and vomiting during the surgery, and 34 patients (16.59\%) in the early postoperative period.

Also, we can extrapolate, as might be anticipated, that the super morbidly obese (BMI $\geq 50$ ) parturients seemed to be more challenging to manage than the morbidly obese parturients (BMI 40-49.9). They had more attempts at needle placement for regional anesthesia [SMO vs. MO number of attempts was 2.6 vs. 2.0 (p-value 0.0255)], more fluids administered (likely to prevent intra-operative hypotension and/or because of the length of the procedure) and required longer times to complete the surgery [SMO vs. MO mean surgical time was $107.55 \mathrm{~min}$ vs. $86.6 \mathrm{~min}$ (p-value 0.0001)]. The implications here are twofold. Higher grades of obesity (super-morbid obesity) should be a risk factor for potential perioperative morbidity added to the recognized and mentioned risks of being overweight or obese in pregnancy. Finally, due consideration should be paid pre-operatively to adjusting the anesthetic technique in these patients. It is desirable to avoid the potentially deleterious effects of either adding intravenous narcotic adjuncts (over sedation, apnea, aspiration) or intra-operative conversion to a general anesthetic (difficult airway access in a difficult position, rapid desaturation) in order to keep the patient comfortable for the duration of the surgery. Perhaps an appropriately placed (e.g., upper lumbar or lower thoracic) epidural technique is a more proficient route of anesthesia in these parturients.

In conclusion, our study found that spinal anesthesia continued to be the preferred technique to provide anesthesia for cesarean sections in morbidly obese parturients. It resulted in higher incidence of intraoperative hypotension than other regional techniques. Those women with delivery $\mathrm{BMI} \geq 50$ appeared to be prone to more challenges during cesarean deliveries. This was evidenced by the higher mean surgical times, more placement attempts of regional anesthesia and larger intra-operative IV fluid volumes. Due consideration should be paid to adjusting the anesthetic technique in bariatric parturients. In addition, anesthesia providers should take advantage of interventions and facilities that aid in performing central regional techniques, as these women present a significant challenge to regional access. For example, the use of spine ultrasound, which should be practiced and implemented more often in bariatric parturients in pursuit of performing regional techniques most efficiently. A well-organized, randomized controlled trial could prove beneficial in validating the most profitable anesthetic technique for bariatric parturients that fosters safety for both mother and baby.

The limitation of our study comes from being a retrospective review with missing data in some patients and the inability to randomize patients into specific groups and the lack of controlled group to compare our cohort to it.

\section{References}

1. [No authors listed] (2000) Obesity: preventing and managing the global epidemic. Report of a WHO consultation. World Health Organ Tech Rep Ser 894: i-xii, 1-253.

2. Alanis MC, Villers MS, Law TL, Steadman EM, Robinson CJ (2010) Complications of cesarean delivery in the massively obese parturient. Am J Obstet Gynecol 203: 271.

3. Alanis MC, Goodnight WH, Hill EG, Robinson CJ, Villers MS, et al. (2010) Maternal super-obesity (body mass index $>$ or $=50$ ) and adverse pregnancy outcomes. Acta Obstet Gynecol Scand 89: 924-930.

4. Cedergren M (2004) Maternal morbid obesity and the risk of adverse pregnancy outcome. Obstet Gynecol 103: 219-224.

5. Pan L, Freedman DS, Gillespie C, Park S, Sherry B (2011) Incidences of obesity and extreme obesity among US adults: findings from the 2009 Behavioral Risk Factor Surveillance System. Popul Health Metr 9: 56.

6. Flegal KM, Carroll MD, Kuczmarski RJ, Johnson CL (1998) Overweight and obesity in the United States: prevalence and trends, 1960-1994. Int J Obes Relat Metab Disord 22: 39-47.

7. Flegal KM, Carroll MD, Ogden CL, Curtin LR (2010) Prevalence and trends in obesity among US adults, 1999-2008. JAMA 303: 235-241.

8. Kim SY, Dietz PM, England L, Morrow B, Callaghan WM (2007) Trends in pre-pregnancy obesity in nine states, 1993-2003. Obesity (Silver Spring) 15: 986-993.

9. El-Chaar D, Finkelstein SA, Tu X, Fell DB, Gaudet L, et al. (2013) The impact of increasing obesity class on obstetrical outcomes. J Obstet Gynaecol Can 35: 224-233.

10. Andreasen KR, Andersen ML, Schantz AL (2004) Obesity and pregnancy. Acta Obstet Gynecol Scand 83: 1022-1029. 
11. Broberger C (2005) Brain regulation of food intake and appetite: molecules and networks. J Intern Med 258: 301-327.

12. Unterborn $\mathrm{J}$ (2001) Pulmonary function testing in obesity, pregnancy, and extremes of body habitus. Clin Chest Med 22: 759-767.

13. Dempsey JA, Reddan W, Rankin J, Balke B (1966) Alveolar-arterial gas exchange during muscular work in obesity. J Appl Physiol 21: 1807-1814.

14. Soens MA, Birnbach DJ, Ranasinghe JS, van Zundert A (2008) Obstetric anesthesia for the obese and morbidly obese patient: an ounce of prevention is worth more than a pound of treatment. cta Anaesthesiol Scand 52: 6-19.

15. Veille JC, Hanson R (1994) Obesity, pregnancy, and left ventricular functioning during the third trimester. Am J Obstet Gynecol 171: 980-983.

16. Vasan RS (2003) Cardiac function and obesity. Heart 89: 1127-1129.

17. Tomoda S, Tamura T, Sudo Y, Ogita S (1996) Effects of obesity on pregnan women: maternal hemodynamic change. Am J Perinatol 13: 73-78.

18. Shnaider R, Ezri T, Szmuk P, Larson S, Warters RD, et al. (2001) Combined spinal-epidural anesthesia for Cesarean section in a patient with peripartum dilated cardiomyopathy. Can J Anaesth 48: 681-683.

19. Hood DD, Dewan DM (1993) Anesthetic and obstetric outcome in morbidly obese parturients. Anesthesiology 79: 1210-1218.

20. Roofthooft E (2009) Anesthesia for the morbidly obese parturient. Curr Opin Anaesthesiol 22: 341-346.

21. Weiss JL, Malone FD, Emig D, Ball RH, Nyberg DA, et al. (2004) Obesity, obstetric complications and cesarean delivery rate--a population-based screening study. Am J Obstet Gynecol 190: 1091-1097.
22. Perlow JH, Morgan MA (1994) Massive maternal obesity and perioperative cesarean morbidity. Am J Obstet Gynecol 170: 560-565

23. Juvin P, Lavaut E, Dupont H, Lefevre P, Demetriou M, et al. (2003) Difficult tracheal intubation is more common in obese than in lean patients. Anesth Analg 97: 595-600.

24. Bucklin BA, Hawkins JL, Anderson JR, Ullrich FA (2005) Obstetric anesthesia workforce survey: twenty-year update. Anesthesiology 103: 645-653.

25. Shibli KU, Russell IF (2000) A survey of anaesthetic techniques used for caesarean section in the UK in 1997. Int J Obstet Anesth 9: 160-167.

26. Bamgbade OA, Khalaf WM, Ajai O, Sharma R, Chidambaram V, et al. (2009) Obstetric anaesthesia outcome in obese and non-obese parturients undergoing caesarean delivery: an observational study. Int J Obstet Anesth 18 221-225.

27. Kyokong $\mathrm{O}$, Charuluxananan $\mathrm{S}$, Sriprajittichai $\mathrm{P}$, Poomseetong $\mathrm{T}$, Naksin $\mathrm{P}$ (2006) The incidence and risk factors of hypotension and bradycardia associated with spinal anesthesia. J Med Assoc Thai 89: 58-64.

28. Vricella LK, Louis JM, Mercer BM, Bolden N (2011) Impact of morbid obesity on epidural anesthesia complications in labor. Am J Obstet Gynecol 205: 370.

29. Ohpasanon P, Chinachoti T, Sriswasdi P, Srichu S (2008) Prospective study of hypotension after spinal anesthesia for cesarean section at Siriraj Hospital: incidence and risk factors, Part 2. J Med Assoc Thai 91: 675-680.

30. Roberts SW, Leveno KJ, Sidawi JE, Lucas MJ, Kelly MA (1995) Fetal acidemia associated with regional anesthesia for elective cesarean delivery. Obstet Gynecol 85: 79-83. 